\title{
Liraglutide reduces the body weight and waist circumference in Chinese overweight and obese type 2 diabetic patients
}

Ping FENG ${ }^{1}$, De-min $\mathrm{YU}^{2}$, Li-ming $\mathrm{CHEN}^{2}$, Bao-cheng $\mathrm{CHANG}^{2}$, Qiu-di $\mathrm{JI}^{1}$, Shu-ying $\mathrm{LI}^{1}$, Mei ZHU ${ }^{1}$, Sheng-hua DING ${ }^{3}$, Baozhen ZHANG ${ }^{3}$, Su-li WANG ${ }^{4}$, Hong-tao LI $^{5}$, Jing-na LIN ${ }^{6}$, Mao-jun WANG ${ }^{7}$, Jian-chao GUO ${ }^{8}$, Jie LIU ${ }^{9}$, Zhong-dong LIU ${ }^{9}$, Shentao $\mathrm{WU}^{10}$, Ju-hong $\mathrm{YANG}^{2,}{ }^{*}$, Clinical Cooperation Group of Liraglutide in Chinese Type 2 Diabetes

\begin{abstract}
${ }^{1}$ The General Hospital of Tianjin Medical University, Tianjin 300052, China; ${ }^{2} 2011$ Collaborative Innovation Center of Tianjin for Medical Epigenetics, Key Laboratory of Hormone and Development (Ministry of Health), Metabolic Disease Hospital \& Tianjin Institute of Endocrinology, Tianjin Medical University, Tianjin 300070, China; ${ }^{3}$ The Chinese PLA464 Hospital, Tianjin 300381, China; ${ }^{4}$ Department of Endocrinology, the Affiliated Hospital of Logistics University of Chinese People's Armed Police Forces, Tianjin 300162, China; ${ }^{5}$ Armed Police Crops Hospital, Tianjin 300162, China; ${ }^{6}$ Tianjin People's Hospital, Tianjin 300121, China; ${ }^{7}$ Tian Jin Shi Xian Shui Gu Hospital, Tianjin 300052, China; ${ }^{8}$ The Second Hospital of Tianjin Medical University, Tianjin 300211, China; ${ }^{9}$ Nankai Hospital of Traditional Chinese Medicine, Affiliated with Tianjin University of Traditional Chinese Medicine, Tianjin 300102, China; ${ }^{10}$ The First Hospital Affiliated to Tianjin Medical University, Tianjin 300193, China
\end{abstract}

Aim: To investigate the effects of liraglutide, a glucagon-like peptide-1 (GLP-1) receptor activator, on body weight and waist circumference in Chinese overweight and obese type 2 diabetic patients.

Methods: A total of 328 Chinese overweight and obese type 2 diabetic patients were included in this multi-center, open-labeled and self-controlled clinical study. The patients were subcutaneously injected with liraglutide once daily for 24 weeks as add-on therapy to their previous hypoglycemic treatments. Statistical analyses were performed using SPSS software package version 11.5 for Windows. Results: Liraglutide treatment caused significant reduction of the mean body weight (from $86.61 \pm 14.09$ to $79.10 \pm 13.55 \mathrm{~kg}$ ) and waist circumference (from $101.81 \pm 13.96$ to $94.29 \pm 14.17 \mathrm{~cm}$ ), resulting in body weight lose of $5 \%-10 \%$ in $43.67 \%$ patients, and body weight loss above $10 \%$ in $34.06 \%$ patients, who had significant lower plasma creatinine levels. Baseline waist circumference, BMI and HOMA-IR were independently correlated with the body weight loss. Furthermore, liraglutide treatment significantly decreased HbA1c levels (from $8.66 \% \pm 2.17 \%$ to $6.92 \% \pm 0.95 \%$ ) with $\mathrm{HbA} 1 \mathrm{c}<7.0 \%$ in $35.37 \%$ patients, who had a significantly lower baseline level of $\mathrm{HbA1c}$, but higher baseline levels of $\mathrm{C}$ peptide and glucagon. Moreover, liraglutide treatment resulted in greater body weight loss in patients with a long duration of diabetes, and better glycemic control in patients with a short duration of diabetes.

Conclusion: Liraglutide significantly reduces body weight and waist circumference in Chinese overweight and obese type 2 diabetic patients. Patients with apparent visceral obesity, insulin resistance and a long duration of diabetes may have greater body weight loss; whereas patients with high insulin-secreting ability, hyperglucagonemia, and short-duration diabetes may obtain better glycemic control with liraglutide.

Keywords: liraglutide; diabetes; Chinese type 2 diabetic patients; obesity; body weight; waist circumference; creatinine; HbA1c; C peptide; glucagon

Acta Pharmacologica Sinica (2015) 36: 200-208; doi: 10.1038/aps.2014.136; published online 26 Jan 2015

\section{Introduction}

Type 2 diabetes is characterized by insulin resistance and progressive $\beta$-cell failure. Treatment often must be intensified over time, usually by a combination of agents that address

\footnotetext{
* To whom correspondence should be addressed.

E-mail megii0315@126.com

Received 2014-05-28 Accepted 2014-11-18
}

both insulin resistance and $\beta$-cell dysfunction ${ }^{[1,2]}$. However, several available therapies increase the risk for hypoglycemia and weight gain, which may reduce adherence and lead to poor glycemic control ${ }^{[3]}$. Glucagon-like peptide-1 (GLP-1)based therapies, such as GLP-1 receptor activators, stimulate insulin secretion and reduce glucagon secretion only during hyperglycemia. Glucagon-like peptide-1 receptor activators also slow gastric emptying and reduce appetite ${ }^{[4-6]}$. Liraglu- 
tide, a GLP-1 receptor activator, has proven to be efficient for glycemic control in type 2 diabetic patients both in China and throughout the world ${ }^{[7,8]}$. Liraglutide is effective in patients with maturity-onset diabetes of the young (MODY) 3, and produces lower hypoglycemic risk than glimepiride ${ }^{[9]}$. Moreover, liraglutide has also been used as an anti-obesity therapy ${ }^{[10]}$. In addition, liraglutide is currently applied in the treatment of other insulin resistance-related diseases. In one study, researchers found that short-term combination treatment of liraglutide and metformin was associated with significant weight loss and a decrease in waist circumference in obese women with polycystic ovary syndrome who had previously been poor responders regarding weight reduction while on metformin monotherapy ${ }^{[11]}$. Another pilot study demonstrated that treatment with liraglutide had a good safety profile and significantly improved liver function and histological features in non-alcoholic steatohepatitis patients with glucose intolerance $^{[12]}$. In a recent study, Dutch researchers found that in patients with pronounced insulin-associated weight gain, the addition of liraglutide to their treatment regimen reversed weight gain, decreased insulin dose and improved glycemic control $^{[13]}$. However, except for two studies with small cohort numbers ${ }^{[14,15]}$, there have been no large investigations into the effects of liraglutide on body weight and waist to hip ratio in Chinese obese type 2 diabetic patients. Moreover, the efficacy of liraglutide may be variable in patients of different ethnicities. In this study, we analyzed the effects of liraglutide on body weight and waist circumference in Chinese overweight and obese type 2 diabetic patients. In addition, we analyzed the characteristics of patients with greater weight loss and better glycemic control to determine improved clinical guidelines for care.

\section{Patients and methods Study design}

This was a multi-center, open-labeled and self-controlled clinical study that included 43 centers in China. The Ethics Committee of the University Hospital at Tianjin approved the protocol, and the study was conducted according to the Declaration of Helsinki and the principles of Good Clinical Practice. All patients provided written informed consent before study entry.

Inclusion criteria: (1) Type 2 diabetes (WHO, 1999); (2) aged 18-75 years; (3) BMI $\geq 24 \mathrm{~kg} / \mathrm{m}^{2}[16]$; (4) $\mathrm{HbA1c}<9.0 \%$; (5) without clinically significant cardiac, hepatic, renal or central nervous system diseases; and (6) naïve to hypoglycemic agents or receiving treatment with hypoglycemic agents or insulin for at least 3 months and stable for more than 1 month. According to the diagnostic criteria proposed by the Working Group on Obesity in China, overweight was diagnosed when BMI is $\geq 24$ $\mathrm{kg} / \mathrm{m}^{2}$, and obesity was diagnosed when BMI is $\geq 28 \mathrm{~kg} / \mathrm{m}^{2[16]}$.

Exclusion criteria: (1) women who were pregnant or planning to become pregnant, or were breastfeeding; (2) abnormal liver function with ALT greater than 2.5 times the normal range; (3) abnormal kidney function with blood creatinine $\geq 133 \mu \mathrm{mol} / \mathrm{L}$; (4) hypersensitivity to liraglutide; (5) use of non-diabetic agents affecting glycemic control; (6) use of systemic glucocorticoids or weight loss drugs; (7) acute diabetic complication, infection, etc; (8) other endocrine diseases; (9) a history of pancreatitis, cancer of the pancreas, type 2 multiple endocrine neoplasia syndrome (MENS), or medullary thyroid carcinoma; and (10) inflammatory intestinal diseases or diabetic gastric paretitis.

Intervention: The study included 3 phases. Phase 1 was the screening phase (week -2 to week 0 ), during which patients meeting the inclusion criteria were enrolled into the study and continued their current life style interventions and hypoglycemic therapy for 2 weeks. Phase 2 was the titration phase (week 0 to week 6), during which treatment with $0.6 \mathrm{mg}$ liraglutide was initiated in all patients. The dose was then increased to $1.2 \mathrm{mg}$ or $1.8 \mathrm{mg}$, according to the fasting blood glucose (FBG) level, in 2 week intervals. The dose was then maintained at $1.2 \mathrm{mg}$ or $1.8 \mathrm{mg}$ during the study. Phase 3 was the maintenance phase (week 7 to week 24).

Liraglutide was injected subcutaneously once daily in the upper arm, abdomen, or thigh using a pen injector device. The patients were encouraged to inject liraglutide at the same time each day.

Primary endpoints were changes in body weight, BMI, waist circumference, and the ratio of waist circumference to height (WHR). Secondary endpoints included changes in HbA1c, fasting and postprandial blood glucose, changes in islet function, and changes in blood pressure. Hypoglycemia and other adverse drug reactions were also recorded. The participants were asked to test glucose levels whenever they had symptoms that might be related to hypoglycemia. Biochemical hypoglycemia was defined as a documented glucose level of less than $3.9 \mathrm{mmol} / \mathrm{L}$. Severe hypoglycemia was defined as symptoms consistent with hypoglycemia, requiring the assistance of another person and that was associated with either a glucose level of less than $3.1 \mathrm{mmol} / \mathrm{L}$ or prompt recovery after administration of oral carbohydrate, intravenous glucose, or glucagon. Nocturnal hypoglycemia was defined as occurring after the bedtime injection and before the participant awoke in the morning.

\section{Statistical analysis}

Statistical analyses were performed using SPSS software package version 11.5 for Windows. Continuous variables are presented as the mean and standard deviation (SD). Differences among groups were evaluated using the Kruskal-Wallis H test. Differences before and after treatment were analyzed using the Wilcoxon signed rank sum test. Differences among groups for categorical data were evaluated using the chi-square test. Differences before and after treatment were analyzed using McNemar's test. A P-value of less than 0.05 (2-tailed test) was considered statistically significant. Multiple linear regression models were used to assess the associations between body weight loss and other variables as predictors. 


\section{Results}

\section{Baseline characteristics}

A total of 328 patients were included, and 241 patients completed the study. Eighty-seven patients withdrew from the study. The reasons for withdrawal were economic reasons (54 cases), adverse events (10 cases), inadequate glycemic control (4 cases), inadequate body weight control (3 cases), loss upon follow-up (9 cases), withdrawal of consent (3 cases), and switching to oral glycemic agents because of the improvement of blood glucose (3 cases). The adverse events leading to withdrawal included nausea, vomiting, and diarrhea, etc.

Statistical analysis was based on intention-to-treat (ITT)

Table 1. Demographic characteristics of study participants.

\begin{tabular}{lcc}
\hline & $n$ & Mean \pm SD \\
\hline Gender & M/F & $238 / 90$ \\
Age & 327 & $47 \pm 11$ \\
Duration (year) & 325 & $4.6 \pm 4.3$ \\
HbA1c $(\%)$ & 328 & $8.7 \pm 2.2$ \\
Body weight $(\mathrm{kg})$ & 328 & $86.6 \pm 14.1$ \\
BMI $\left(\mathrm{kg} / \mathrm{m}^{2}\right)$ & 328 & $29.6 \pm 3.8$ \\
WC $(\mathrm{cm})$ & 327 & $102 \pm 14$ \\
WHR & 327 & $0.60 \pm 0.08$ \\
SBP $(\mathrm{mmHg})$ & 328 & $129 \pm 13$ \\
DBP $(\mathrm{mmHg})$ & 328 & $83 \pm 9$ \\
Heart rate & 328 & $78 \pm 6$ \\
FBG $(\mathrm{mmol} / \mathrm{L})$ & 327 & $8.1 \pm 1.6$ \\
Fasting C peptide $(\mathrm{ng} / \mathrm{mL})$ & 120 & $2.2 \pm 1.6$ \\
Fasting insulin $(\mu \mathrm{IU} / \mathrm{L})$ & 98 & $15.6 \pm 10.5$ \\
Fasting glucagon $(\mathrm{pg} / \mathrm{mL})$ & 31 & $139.2 \pm 133.5$ \\
\hline
\end{tabular}

Note: FBG, Fasting blood glucose; WHR, ratio of waist circumference to height; WC, waist circumference; BMI, body mass index. analysis including all 328 patients. The mean imputation method was used to replace missing values. The characteristics of the participants at baseline are presented in Table 1. Among the 328 patients, 74 patients $(22.56 \%)$ were treated with liraglutide alone, 96 patients $(29.27 \%)$ were treated with liraglutide combined with insulin, 54 patients (16.46\%) were treated with liraglutide combined with insulin secretagogues, and 104 patients $(31.71 \%)$ were treated with liraglutide combined with non-insulin secretagogues (glycosidase inhibitors, thiazolidinedione).

\section{Changes in body weight and WHR}

Except for 3 patients who had a mild body weight increase (increased 3.5, 1.9, and $2 \mathrm{~kg}$ ), all of the other patients showed body weight loss at the end of the study (Figure 1). After 24 weeks of treatment, decreases were observed for mean body weight (from $86.61 \pm 14.09$ to $79.10 \pm 13.55 \mathrm{~kg}$ ), BMI (from $29.55 \pm 3.83$ to $27.75 \pm 9.99 \mathrm{~kg} / \mathrm{m}^{2}$ ), waist circumference (from $101.81 \pm 13.96$ to $94.29 \pm 14.17 \mathrm{~cm}$ ) and WHR (from $0.60 \pm 0.08$ to $0.55 \pm 0.10$ ). The mean decrease in body weight was $7.34 \pm 4.23$ $\mathrm{kg}$ (range: $1-22.00 \mathrm{~kg}$ ). The overall percent change in body weight was $8.47 \% \pm 4.53 \%$ (with a maximum observed value of $22.35 \%$ ). Approximately $22.27 \%, 43.67 \%$, and $34.06 \%$ patients attained the targets of body weight loss of $<5 \%, 5 \%-10 \%$, and $\geq 10 \%$, respectively. The percentage of patients with $\mathrm{BMI}<24$ $\mathrm{kg} / \mathrm{m}^{2}$ increased from $0 \%$ at baseline to $32.32 \%$ by the end of study $(P<0.01)$; the percentage of patients with $\mathrm{BMI} \geq 28$ $\mathrm{kg} / \mathrm{m}^{2}$ decreased from $62 \%$ at baseline to $45.53 \%$ by the end of study $(P<0.01)$. Patients with higher BMI values showed greater body weight loss. In patients with $24 \leq \mathrm{BMI}<28,28 \leq$ $\mathrm{BMI}<30$, or $\mathrm{BMI} \geq 30 \mathrm{~kg} / \mathrm{m}^{2}$, the body weight reductions were $6.27 \pm 3.45,7.28 \pm 3.60$, and $8.67 \pm 5.14 \mathrm{~kg}$, respectively $(P=0.001)$ (Figure 2). After 24 weeks of combination therapy with liraglutide, 99 patients changed from obesity to overweight or
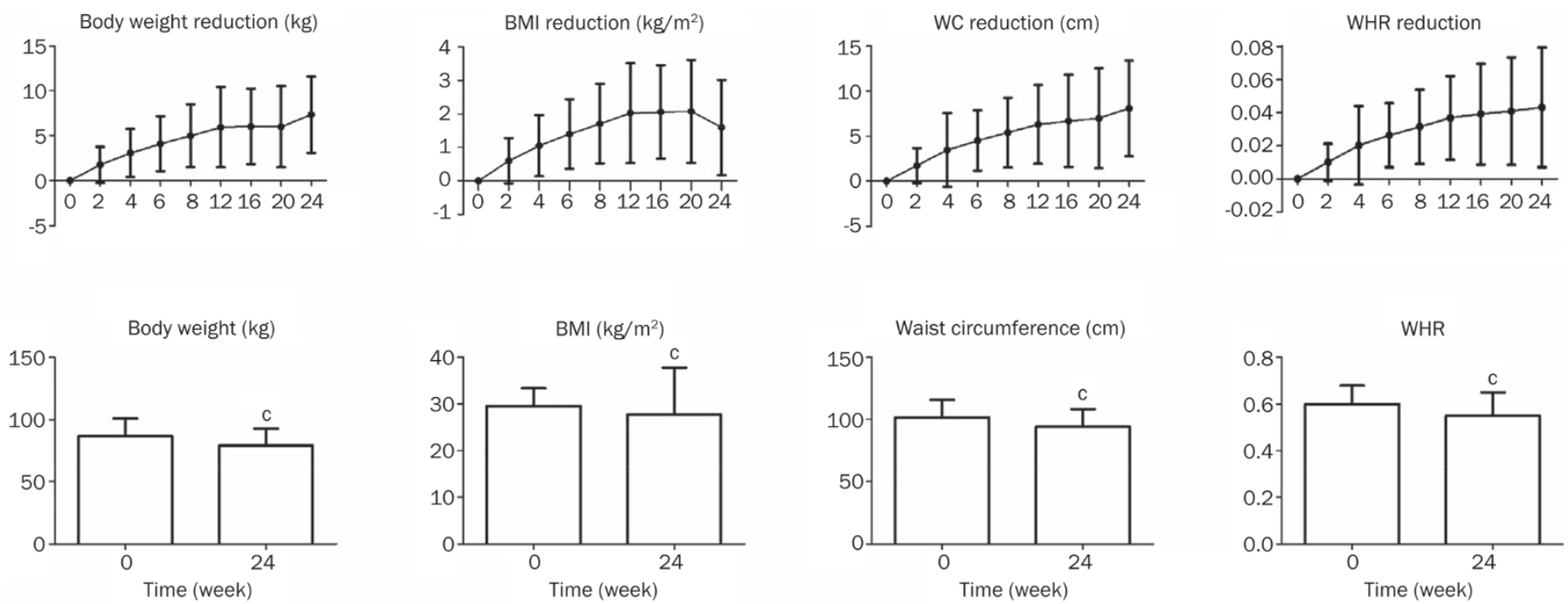

Figure 1. Changes of body weight, waist circumference, BMI and WHR from baseline. Three-hundred and twenty eight patients were given liraglutide on the basis of their previous hypoglycemic medicine for 24 weeks. All the parameters were decreased during the treatment period, and the changes were of statistical significance $(P<0.01)$. BMI, body mass index; WC, waist circumference; WHR, waist and height ratio. 
A

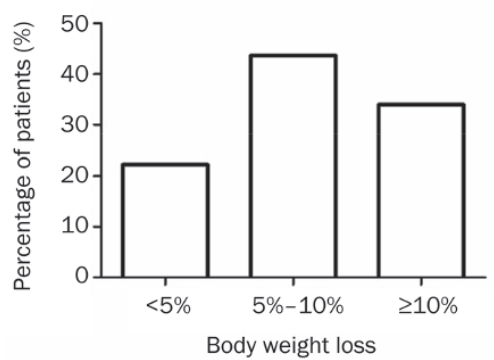

B

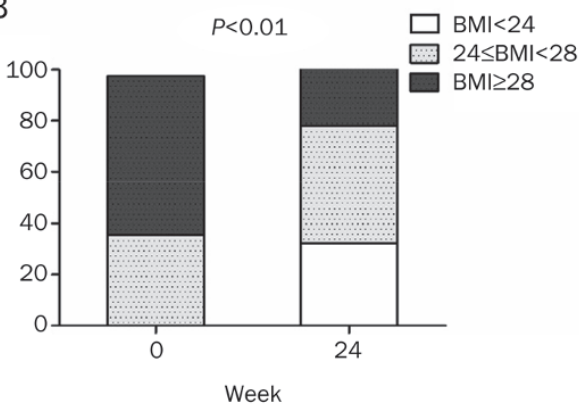

C

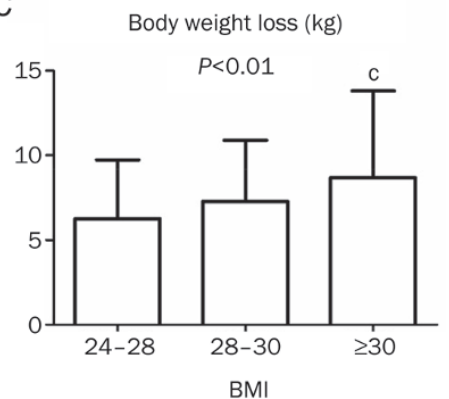

Figure 2. Weight loss in different subgroup of patients. (A) Percentage for patients with body weight loss $<5 \%, 5 \%-10 \%$, and $\geq 10 \%$ at the end of the study. (B) Percentage of patients diagnosed with overweight or obesity at baseline and the end of the study. (C) Weight loss in patients within three baseline BMI catigories. ${ }^{\circ} P<0.01$ compared with patients with $24<\mathrm{BMl}<28 \mathrm{~kg} / \mathrm{m}^{2}$ and $28<\mathrm{BMl}<30 \mathrm{~kg} / \mathrm{m}^{2}$. BMI, body mass index.

from overweight to normal, and another 67 patients changed from obesity to normal. In terms of the body weight- or waist circumference-lowering effects of liraglutide, there was a difference between treatment with 0.6 and 1.2 or $1.8 \mathrm{mg}$, but no difference was found between 1.2 and $1.8 \mathrm{mg}$ (Figure 3).

\section{Characteristics of patients who obtained better weight control}

Waist circumference $(r=0.336)$, WHR $(r=0.323)$, HOMA-IR $(r=0.261)$, BMI $(r=0.225)$, and body weight $(r=0.167)$ at base- line were correlated with body weight loss. Age, concomitant hypoglycemic medicine, duration of diabetes, baseline blood pressure, and fasting plasma $\mathrm{C}$ peptide, insulin, and glucagon levels at baseline were not correlated with body weight loss. Further analysis found that BMI, HOMA-IR and waist circumference at baseline were independent correlated factors for body weight loss (Beta $=0.523,0.212$, and 0.227, respectively, $P<0.05$ ) (Figure 4).

In the patients whose body weight decreased by more than
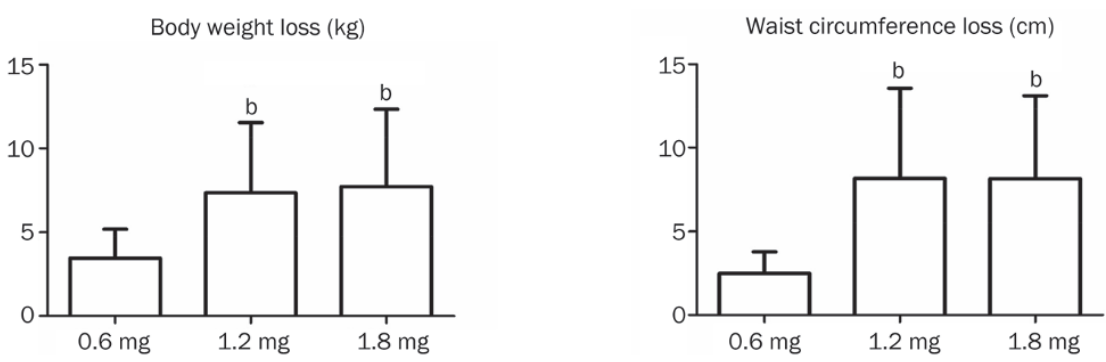

Figure 3. Weight loss in patients using different dosages of liraglutide. The number of patients using $0.6,1.2$, and $1.8 \mathrm{mg}$ of liraglutide were 4,182 , and 41 , respectively. ${ }^{b}$ Compared with patients using $0.6 \mathrm{mg}$ liraglutide $P<0.05$. There was no difference between 1.2 and $1.8 \mathrm{mg}$ liraglutide both in weight loss and in waist circumeference loss.
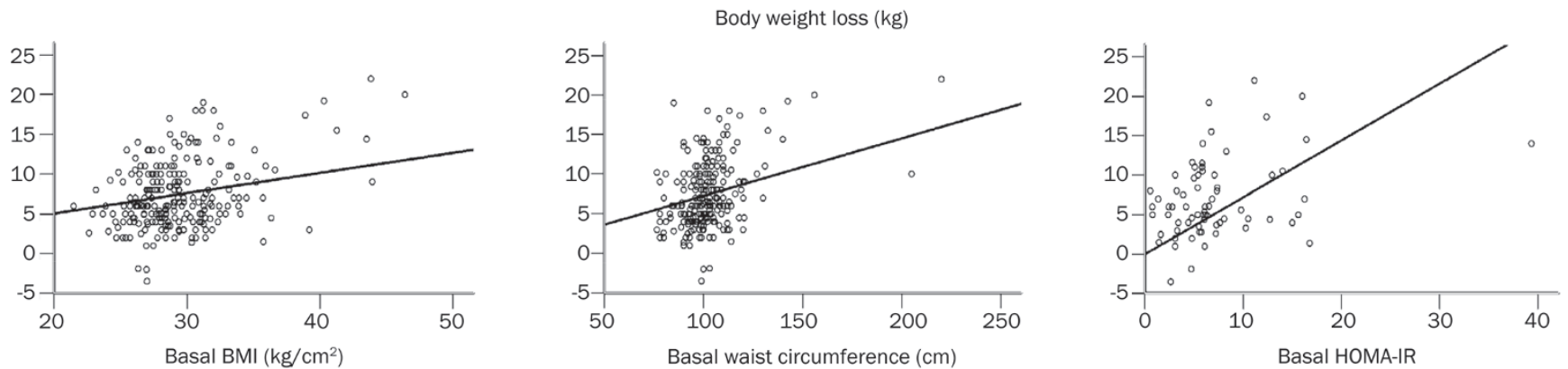

Figure 4. Relationship between baseline BMI, waist circumference, HOMA-IR and body weight reduction at the end of the study. Beta=0.523, 0.212 , and 0.227 , respectively. $P<0.01$. BMI, body mass index; HOMA-IR, HOMA insulin resistance index. Waist circumference, WHR, HOMA-IR, BMI and body weight at baseline were correlated with body weight loss; Multivariate regression analysis incuding body weight loss as dependent variable and waist circumference, WHR, HOMA-IR, BMI as independent variables, only BMI, HOMA-IR and waist circumference at baseline were the independent correlated factors for body weight loss; Age, concomitant hypoglycemic medicine, duration of diabetes, baseline blood pressure, fasting plasma $\mathrm{C}$ peptide, insulin, glucagon level at baseline were not correlated with body weight loss. 
$10 \%$, baseline waist circumference, WHR and HOMA-IR were significantly higher than in the patients whose body weight decreased by less than $10 \%$. Moreover, the patients whose body weight decreased by more than $10 \%$ had lower levels of plasma creatinine than the patients whose body weight decreased by no more than $10 \%$. Other factors such as blood lipid level, duration of diabetes, blood pressure and baseline fasting $C$ peptide and glucagon levels were not correlated with body weight loss (Figure 5).

After 24 weeks of treatment, body weight and waist circumference decreased to a greater extent in the patients with a longer duration of diabetes. In the patients with a disease duration of longer than 10 years, liraglutide treatment led to the most significant decrease in body weight and waist circumference $(P<0.05$, Figure 6$)$.

\section{Changes in blood glucose}

During the study, both fasting blood glucose and postprandial blood glucose decreased continuously (Figure 7). After 24 weeks of treatment, $\mathrm{HbA1c}$ decreased from $8.66 \% \pm 2.17 \%$ to $6.92 \% \pm 0.95 \% \quad(P<0.05)$. The percentage of patients attaining $\mathrm{HbA} 1 \mathrm{c}<7 \%$ changed from $8.54 \%(28 / 328)$ at baseline to $35.37 \%$ $(116 / 227)$ at the 24 th week. The percentage of patients attaining $\mathrm{HbA} 1 \mathrm{c}<6.5 \%$ changed from $1.52 \%(5 / 328)$ at baseline to $16.77 \%(55 / 227)$ at the 24 th week.
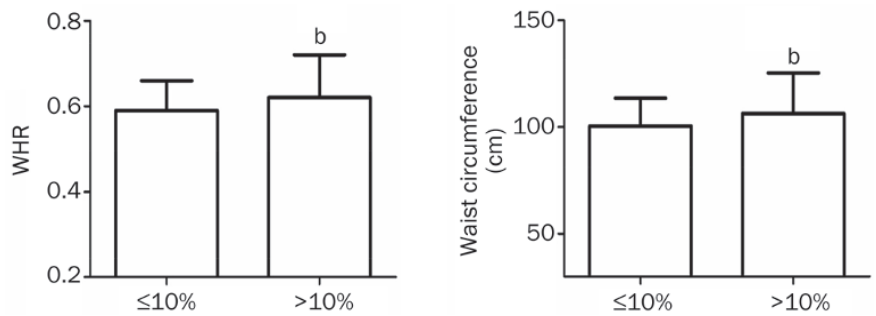

\section{Characteristics of the patients who obtained glycemic control}

Compared with the patients with $\mathrm{HbA} 1 \mathrm{c} \geq 7 \%$, the patients with $\mathrm{HbA1c}<7 \%$ had higher levels of baseline fasting $\mathrm{C}$ peptide and glucagon but a lower level of HbA1c (Figure 8). Other characteristics including age, body weight, waist circumference, fasting insulin level, and creatinine level were comparable between the groups.

\section{Islet function and insulin resistance}

After 24 weeks of liraglutide treatment, there were no significant changes in the levels of $C$ peptide $(1.92 \pm 1.11$ vs $2.13 \pm 2.49$ $\mathrm{ng} / \mathrm{mL}, P=0.48)$, insulin $(17.62 \pm 11.32$ vs $18.10 \pm 10.93 \mu \mathrm{IU} / \mathrm{L}$, $P=0.06)$, or glucagon $(206.52 \pm 182.75$ vs $174.46 \pm 112.45 \mathrm{pg} / \mathrm{mL}$, $P=0.14)$; but HOMA-IR was significantly improved (5.30 \pm 4.24 vs $4.31 \pm 4.09, P<0.01$ ) (Figure 9). The insulin dosage decreased from $0.45 \pm 0.25 \mathrm{IU} /(\mathrm{kg} \cdot \mathrm{d})$ at baseline to $0.33 \pm 0.21 \mathrm{IU} /(\mathrm{kg} \cdot \mathrm{d})$ at week 24 .

\section{Hypoglycemia and other adverse events}

During the treatment period, 24 patients experienced 34 hypoglycemic episodes. No severe hypoglycemia was reported. Among the patients experiencing hypoglycemia, 10 patients were treated with insulin (9 patients with premixed insulin, 1 patient with basal insulin), 10 patients were treated with insulin secretagogues (6 patients with glimepiride, 4 patients
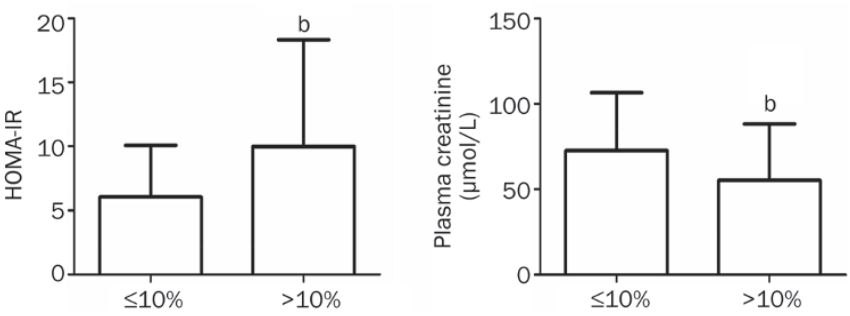

Figure 5. Characteristics of patients with body weight reduction greater than $10 \%$. Patients with body weight decreased more than $10 \%$ had significantly higher level of baseline waist circumference, baseline WHR and HOMA-IR and lower level of plasma creatinine compared with those with body weight decreased less than $10 \%$. Other factors such as age, diabetic duration, HbA1c, body weight were not different between groups. ${ }^{b} P<0.05$ compared with patients with body weight decreased $<10 \%$.

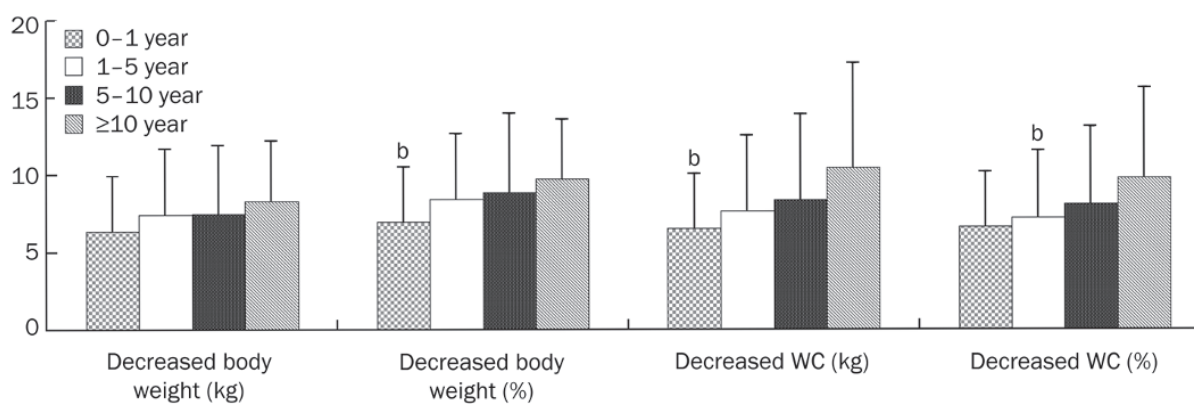

Figure 6. Association between duration of diabetes and change in body weight, waist circumference at end of the study. After 24 weeks of treatment, body weight and waist circumference decreased greater in patients with longer duration of diabetes. Other characteristics including age, body weight, waist circumference, fasting insulin, creatinine were comparable between patients with and without $\mathrm{HbA} 1 \mathrm{c}<7 \%$. ${ }^{\mathrm{b}} \mathrm{P}<0.05$ compared with patients with duration of diabetes longer than 10 year. 

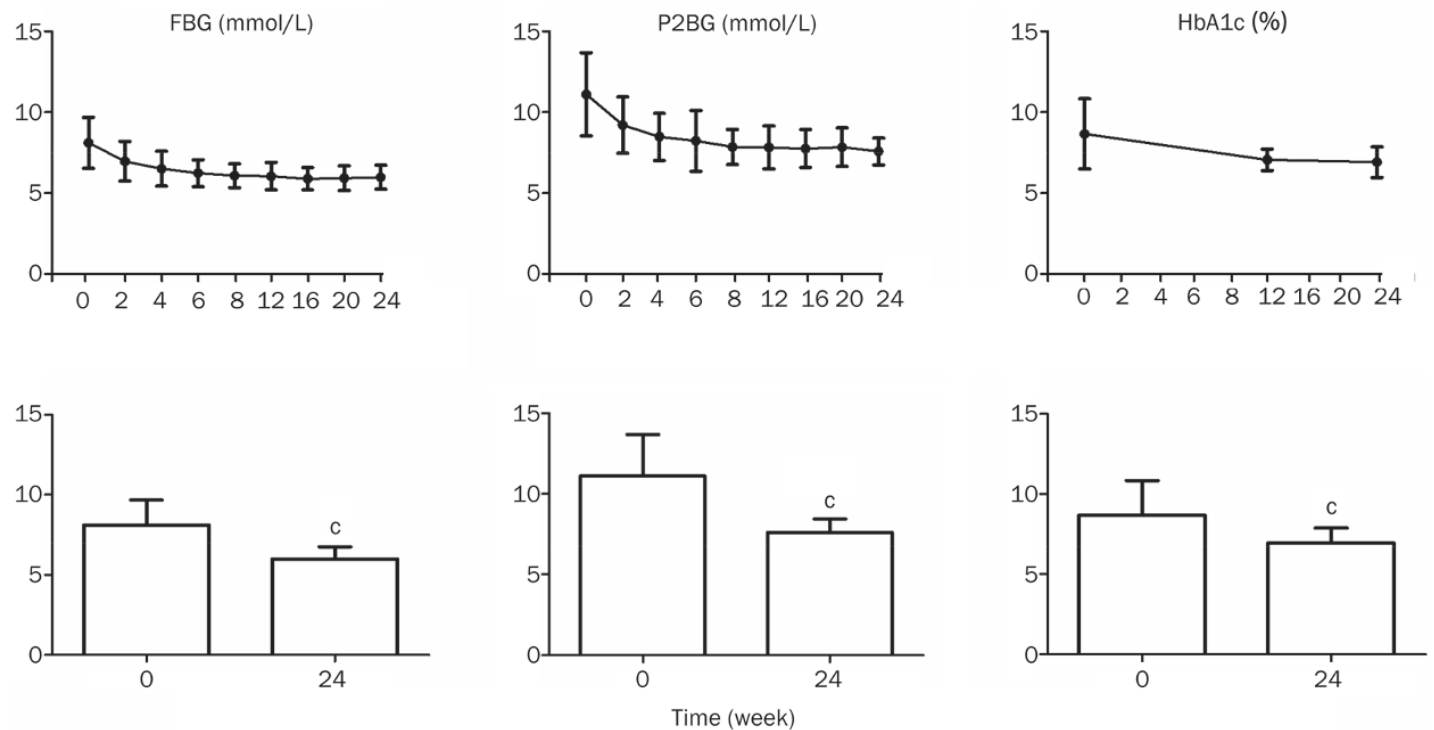

Figure 7. Blood glucose before and after liraglutide treatment. FBG, fasting blood glucose; $P 2 B G$, postprandial blood glucose. ${ }^{\circ} P<0.01$ compared with baseline.
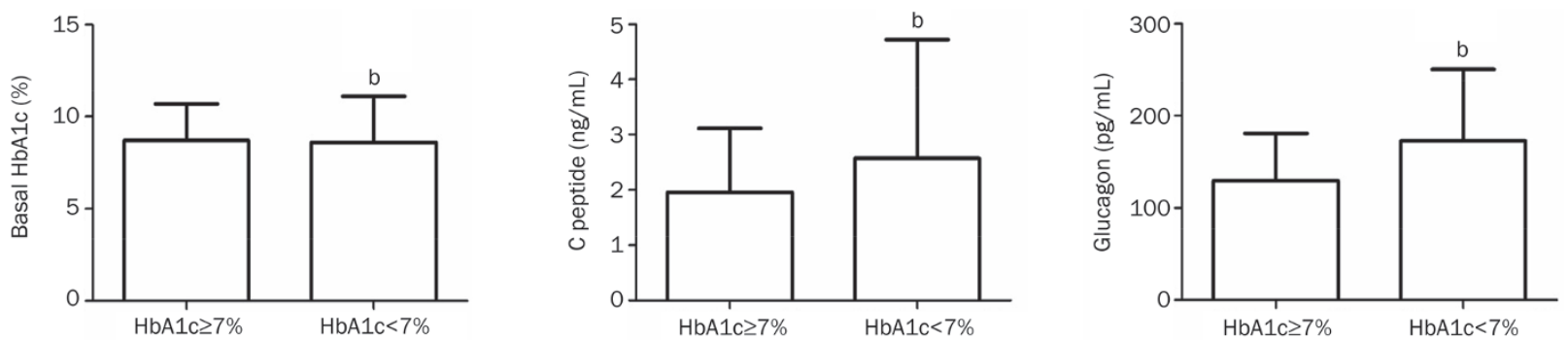

Figure 8. Characteristics of patients with $\mathrm{HbA} 1 \mathrm{c}<7 \%$ after liraglutide treatment. Compared with patients with $\mathrm{HbA} 1 \mathrm{c} \geq 7 \%$, patients with $\mathrm{HbA} 1 \mathrm{c}<7 \%$ had a higher level of fasting $\mathrm{C}$ peptide and fasting glucagon, but a lower level of $\mathrm{HbA} 1 \mathrm{C}$ at baseline. Other characteristics including age, body weight, waist circumference, fasting insulin, creatinine were comparable between the two groups of patients. ${ }^{b} P<0.05$ compared with $\mathrm{HbA} 1 \mathrm{c} \geq 7 \%$.
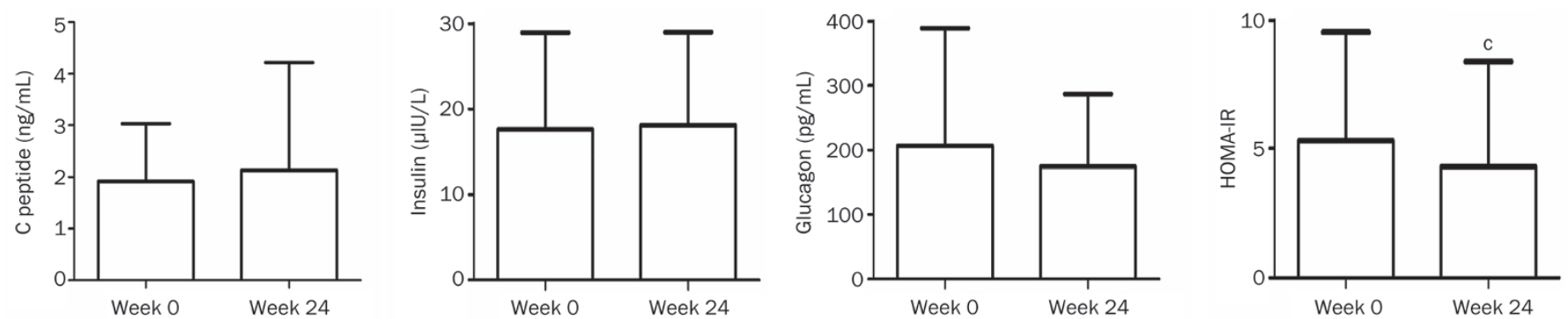

Figure 9. Change in islet function and insulin resistance after 24 weeks of liraglutide treatment. After 24 weeks of treatment, HOMA-IR was improved significantly $(5.30 \pm 4.24$ vs $4.31 \pm 4.09, P=0.00)$, while the changes in $C$ peptide $(P=0.48)$, insulin $(P=0.06)$, and glucagon $(P=0.14)$ did not reach significant level. ${ }^{\circ} P<0.01$ compared with baseline.

with repaglinide), 3 patients were treated with metformin, and 1 patient was treated with thiazolidinedione and voglibose. Among these patients, 2 withdrew from the study because of serious gastrointestinal side effects such as nausea, vomiting and diarrhea at the 2nd week; and 1 withdrew from the study because of decreased orexis.

During the 24 weeks, 45 patients $(14.85 \%)$ experienced gastrointestinal symptoms such as abdominal distention, nausea, diarrhea, and decreased orexis. Gastrointestinal symptoms occurred more frequently during the first 2 weeks and 
included nausea and vomiting (6.27\%), anepithymia (3.63\%), diarrhea $(1.32 \%)$, abdominal distention $(2.64 \%)$ and constipation $(0.99 \%)$. All side effects decreased markedly after 4 weeks $(2.46 \%, 1.75 \%, 1.40 \%, 1.40 \%, 0.35 \%$, respectively), and decreased to less than $1 \%$ after 8 weeks.

\section{Discussion}

GLP-1 receptor activators are increasingly used as secondor third-line therapies in type 2 diabetic patients after failure of metformin monotherapy or in combination with other anti-diabetic medications ${ }^{[17]}$. In addition to being effective in improving glucose control by reducing $\mathrm{HbA1c}$, therapy with GLP-1 receptor activators such as liraglutide also induces significant weight $\operatorname{loss}^{[18,19]}$, which is a desirable effect in overweight and obese type 2 diabetic patients. This provides additional benefits in terms of slowing disease progression and improving the general cardiovascular risk profile ${ }^{[20]}$.

In our study, most patients obtained various degrees of weight loss after liraglutide treatment for 24 weeks. The mean decrease in body weight was $7.34 \pm 4.23 \mathrm{~kg}$, and the percentage of body weight loss was $8.47 \% \pm 4.53 \%$. Surprisingly, the body weight reduction in this study is higher than those in other studies. In another study of Chinese type 2 diabetic patients, liraglutide at a dose of 1.2 or $1.8 \mathrm{mg}$ was associated with a $1.8-2.4 \mathrm{~kg}$ weight reduction. In that study, the baseline body weight of patients was $68.1 \pm 11.7 \mathrm{~kg}$, which was significantly lower than that in our groups $(86.61 \pm 14.09 \mathrm{~kg})^{[8]}$. As proved in our study, the body weight decreased more in patients with higher baseline BMI and body weight. Therefore, it is reasonable to conclude that liraglutide is more potent in patients with higher body weight or BMI. The results in the present study are also inconsistent with studies conducted in Western countries with respect to body weight loss. In patients with BMI greater than $28 \mathrm{~kg} / \mathrm{m}^{2}$, the mean body weight reduction was $1.5-3.3 \mathrm{~kg}$, which is much lower than in our study ${ }^{[21-26]}$. We suspect the substantial decrease of body weight in our study cannot be explained solely by higher baseline BMI. In another study, investigators found that at the same BMI values, Asian patients have a higher body fat composition than patients in Western countries ${ }^{[27]}$. In another study, investigators compared the abdominal fat distribution of Japanese $(n=239)$ and Caucasian-Americans $(n=177)$ aged $40-49$ years, and found that Japanese men had more visceral adipose tissue at the same waist circumference. Therefore, we believe that Chinese patients have a higher level of body fat at the same BMI values and waist circumferences, which might explain why the patients in our study obtained greater weight loss than patients in Western countries. Waist circumference was also decreased to a greater extent in our group (from 101.81 \pm 13.96 to $94.29 \pm 14.17 \mathrm{~cm}$ ) than in other groups (decreased 1.8-4.9 $\mathrm{cm}$ ) in Western countries. Waist circumference is a marker of visceral obesity. In the HOPE study, the investigators found that after 4.5 years of follow up, patients in the third tertile of waist circumference had an increased adjusted RR of $23 \%$ for myocardial infarction, 38\% for heart failure, and 17\% for total mortality ${ }^{[28]}$. Therefore, we speculate that liraglutide may have long-term cardiovascular-protective roles for Chinese obese type 2 diabetic patients through effective weight control and waist circumference improvement.

Not all patients obtained weight loss in our study. A total of 3 patients had a weight increase, and approximately $25 \%$ of patients did not obtain the target of weight control (defined as a decrease of 5\%). In our study, waist circumference, HOMAIR and BMI were independent predisposing factors for body weight loss. Considering the high cost of liraglutide, we conclude that it is more efficient in patients with higher waist circumference and insulin resistance. Fadini et a ${ }^{[29]}$ found that in type 2 diabetic Italian patients, the only independent determinant of weight loss was baseline BMI, which is different from the present study. The reason for the difference is not clear. The difference in ethnicity may be a reason. Moreover, the sample size of our subjects is greater than that in Fadini's study, which might be another reason for this difference.

Moreover, we found that in patients with greater weight loss $(>10 \%)$, baseline plasma creatinine was lower than that in patients with a lower decrease in body weight $(55.32 \pm 32.68$ vs $72.55 \pm 33.94 \mu \mathrm{mol} / \mathrm{L}$ ). For both groups, the average plasma creatinine was in the normal range, which apparently did not reflect the effects of kidney function. Moreover, the serum urea nitrogen was not different between the two groups (6.79 \pm 6.99 vs $6.34 \pm 8.85 \mathrm{mmol} / \mathrm{L})$. Therefore, diet could not explain the difference in plasma creatinine level between the two groups. However, creatinine is also a marker of muscle mass ${ }^{[30]}$; and therefore we believe that liraglutide may bring greater weight loss in patients with less muscle mass. At the same BMI level, patients with less muscle mass have more fat mass. Therefore, our results further support that liraglutide is more effective at reducing body weight in patients with greater waist circumferences and HOMA-IR values. Plasma creatinine may be a useful marker for patients who will obtain greater weight loss results from treatment with liraglutide.

In our study, liraglutide treatment also led to significant improvements in HOMA-IR in type 2 diabetic patients. Insulin dosage decreased from $0.45 \pm 0.25 \mathrm{IU} /(\mathrm{kg} \cdot \mathrm{d})$ at baseline to $(0.33 \pm 0.21) \mathrm{IU} /(\mathrm{kg} \cdot \mathrm{d})$ at week 24 . A decrease in body weight might be a reason for the improvement in insulin resistance. However, in our study, we also found that liraglutide treatment led to a significant reduction in waist circumference $(8.08 \pm 5.31 \mathrm{~cm})$. Waist circumference is a marker of visceral obesity $^{[31]}$. In a study by Jendle et al, a significant loss of visceral fat was found after liraglutide therapy ${ }^{[32]}$. Therefore, liraglutide may improve insulin resistance by lowering visceral obesity.

In the LEAD 1 study, $42 \%$ and $21 \%$ of subjects treated with liraglutide at 1.8 or $1.2 \mathrm{mg}$ for 26 weeks reached $\mathrm{HbA1c}<7.0 \%$ and $\leq 6.5 \%$, respectively. In our study, $\mathrm{HbA1c}$ decreased from $8.66 \% \pm 2.17 \%$ to $6.92 \% \pm 0.95 \%$ after 24 weeks of combination therapy with liraglutide $(P<0.05)$. Approximately $35.37 \%$ obtained $\mathrm{HbA} 1 \mathrm{c}$ levels of $<7 \%$, and $16.77 \%$ obtained the target of $\mathrm{HbA} 1 \mathrm{c}<6.5 \%$. Therefore, liraglutide is not efficient in every patient to establish glycemic control. The patients who attained the target level for glycemic control (HbA1c $<7 \%)$ 
had higher baseline levels of $C$ peptide and glucagon than their counterparts. Therefore, we conclude that liraglutide is more efficient in patients with a higher level of insulinsecreting function and hyperglucagonemia. Our result is in concordance with other studies, which suggested that liraglutide should be recommended to type 2 diabetic patients in the early phase of the disease, when there is still sufficient insulin secretion. Therefore, higher levels of $\mathrm{C}$ peptide and glucagon may be useful markers for patients who will potentially obtain better glycemic control. In our study, we did not find a significant reduction in glucagon levels before and after liraglutide treatment. We also conducted a subgroup analysis in patients with different durations of type 2 diabetes but still did not find any differences in glucagon either in newly diagnosed patients, with a disease duration of less than 3 years, or in patients with a duration of greater than 10 years. However, in the LEAD 3 study, a significant decrease was noticed after liraglutide treatment in newly diagnosed diabetic patients ${ }^{[33]}$. Considering the large variation in glucagon levels between patients in our study, a large study is needed to clarify the effects of liraglutide on glucagon secretion in obese Chinese type 2 diabetes patients. In this study, we did find a difference between the doses of 0.6 and 1.2 or $1.8 \mathrm{mg}$, but we did not find a difference between the doses of 1.2 and $1.8 \mathrm{mg}$ in terms of body weight-lowering effects of liraglutide. However, there were only 4 cases using $0.6 \mathrm{mg}$ in our study; therefore, it is unclear whether 1.2 or $1.8 \mathrm{mg}$ is superior to $0.6 \mathrm{mg}$. On the other hand, from the aspect of body weight control, $1.2 \mathrm{mg}$ may be a suitable dosage to minimize side effects and decrease cost.

However, our study has some limitations. First, we used the ITT method to analyze all the patients to maintain the sample size and to avoid overoptimistic estimates of the efficacy of an intervention ${ }^{[34]}$, but ITT analysis does have some limitations ${ }^{[35]}$. Moreover, we did not include a control group without liraglutide therapy; this attenuates the stringency of the results concerning the weight-loss and glucose-lowering effects of liraglutide.

In conclusion, liraglutide is more effective at controlling body weight and waist circumference for Chinese overweight and obese type 2 diabetic patients than at controlling that of patients in Western countries. Patients with greater BMI, waist circumferences, insulin resistance, long durations of diabetes, and low creatinine levels may obtain greater weight loss; patients with higher $C$ peptide and glucagon levels and short durations of diabetes may obtain greater glycemic control.

\section{Abbreviations}

BMI, body mass index; HbA1c, Glycated hemoglobin A1c; GLP-1, glucagon-like peptide 1; HOMA-IR, homeostatic model assessment index of insulin resistance; WHR, waist circumference to height; FBG, fasting blood glucose

\section{Acknowledgements}

We acknowledge the assistance of investigators and all sub- jects for their participation in this study. This work was supported by the National Natural Science Foundation of China (No 81200612) and the Tianjin City High School Science \& Technology Fund Planning Project (№ 20102217). We also acknowledge Allen Clermont from the Joslin Diabetes Center of Harvard Medical School for assistance in the writing.

\section{Author contribution}

Ping FENG conceived and performed of the study. Ju-hong YANG acquired and analyzed the data and wrote the manuscript. All the other authors acquired and researched the data. All authors read and approved the final manuscript.

\section{References}

1 Kahn SE, Haffner SM, Heise MA, Herman WH, Holman RR, Jones NP, et al. Glycemic durability of rosiglitazone, metformin, or glyburide monotherapy. N Engl J Med 2006; 355: 2427-43.

2 UKPDS Group. Intensive blood-glucose control with sulphonylureas or insulin compared with conventional treatment and risk of complications in patients with type 2 diabetes (UKPDS 33). UK Prospective Diabetes Study (UKPDS) Group. Lancet 1998; 352: 837-53.

3 Green J, Feinglos M. Update on type 2 diabetes mellitus: understanding changes in the diabetes treatment paradigm. Int J Clin Pract 2007; 61 (Suppl 154): 3-11.

4 Holst JJ. The physiology of glucagon-like peptide 1. Physiol Rev 2007; 87: 1409-39.

5 Knudsen LB, Nielsen PF, Huusfeldt PO, Johansen NL, Madsen K, Pedersen FZ. Potent derivatives of glucagon-like peptide-1 with pharmacokinetic properties suitable for once daily administration. J Med Chem 2000; 43: 1664-9.

6 Agerso H, Jensen LB, Elbrond B, Rolan P, Zdravkovic M. The pharmacokinetics, pharmacodynamics, safety and tolerability of NN2211, a new long-acting GLP-1 derivative, in healthy men. Diabetologia 2002; 45: 195-202.

7 Nauck M, Frid A, Hermansen K, Thomsen AB, During M, Shah N, et al. Long-term efficacy and safety comparison of liraglutide, glimepiride and placebo, all in combination with metformin in type 2 diabetes: 2-year results from the LEAD-2 study. Diabetes Obes Metab 2013; 15 : 204-12.

8 Yang W, Chen L, Ji Q, Liu X, Ma J, Tandon N, et al. Liraglutide provides similar glycaemic control as glimepiride (both in combination with metformin) and reduces body weight and systolic blood pressure in Asian population with type 2 diabetes from China, South Korea and India: a 16-week, randomized, double-blind, active control trial. Diabetes Obes Metab 2011; 13: 81-8.

9 Ostoft SH, Bagger J, Hansen T, Pedersen O, Faber J, Holst J, et al. Glucose-lowering effects and low risk of hypoglycemia in patients with maturity-onset diabetes of the young when treated with a GLP1 receptor agonist: a double-blind, randomized, crossover trial. Diabetes Care 2014; 37: 1797-805.

10 Suzuki D, Toyoda M, Kimura M, Miyauchi M, Yamamoto N, Sato H, et al. Effects of liraglutide, a human glucagon-like peptide-1 analogue, on body weight, body fat area and body fat-related markers in patients with type 2 diabetes mellitus. Intern Med 2013; 52: 1029-34.

11 Jensterle Sever M1, Kocjan T, Pfeifer M, Kravos NA, Janez A. Shortterm combined treatment with liraglutide and metformin leads to significant weight loss in obese women with polycystic ovary syndrome and previous poor response to metformin. Eur J Endocrinol 2014; 170: 451-9.

12 Eguchi Y, Kitajima Y, Hyogo H, Takahashi H, Kojima M, Ono M, et 
2046-55.

al. Pilot study of liraglutide effects in non-alcoholic steatohepatitis and non-alcoholic fatty liver disease with glucose intolerance in Japanese patients (LEAN-J). Hepatol Res 2014 May 4. doi: 10.1111/ hepr.12351. [Epub ahead of print]

13 de Wit HM1, Vervoort GM, Jansen HJ, de Grauw WJ, de Galan BE, Tack CJ. Liraglutide reverses pronounced insulin-associated weight gain, improves glycaemic control and decreases insulin dose in patients with type 2 diabetes: a 26 week, randomised clinical trial (ELEGANT). Diabetologia 2014 Jun 20. [Epub ahead of print]

14 Li CJ, Li J, Zhang QM, Lv L, Chen R, Lv CF, et al. Efficacy and safety comparison between liraglutide as add-on therapy to insulin and insulin dose-increase in Chinese subjects with poorly controlled type 2 diabetes and abdominal obesity. Cardiovasc Diabetol 2012; 11: 142

15 Li CJ, Yu Q, Yu P, Yu TL, Zhang QM, Lu S, Yu DM. Changes in liraglutide-induced body composition are related to modifications in plasma cardiac natriuretic peptides levels in obese type 2 diabetic patients. Cardiovasc Diabetol 2014; 13: 36.

16 Bei-Fan Z; Cooperative meta-analysis group of working group on obesity in China. Predictive values of body mass index and waist circumference for risk factors of certain related diseases in Chinese adults: study on optimal cut-off points of body mass index and waist circumference in Chinese adults. Asia Pac J Clin Nutr 2002; 11: S685-S693.

17 Inzucchi SE, Bergenstal RM, Buse JB. Management of hyperglycaemia in type 2 diabetes: a patient-centered approach. Position statement of the American diabetes association (ADA) and the European association for the study of diabetes (EASD). Diabetologia 2012; 55 : 1577-96.

18 Niswender K, Pi-Sunyer X, Buse J. Weight change with liraglutide and comparator therapies: an analysis of seven phase 3 trials from the liraglutide diabetes development programme. Diabetes Obes Metab 2013; 15: 42-54.

19 Zander M, Madsbad S, Madsen JL, Holst JJ. Effect of 6-week course of glucagon-like peptide 1 on glycaemic control, insulin sensitivity, and beta-cell function in type 2 diabetes: a parallel-group study. Lancet 2002; 359: 824-30.

20 van Genugten RE, Moller-Goede DL, van Raalte DH, Diamant M. Extra-pancreatic effects of incretin-based therapies: potential benefit for cardiovascular-risk management in type 2 diabetes. Diabetes Obes Metab 2013; 15: 593-606.

21 Bode BW, Testa MA, Magwire M, Hale PM, Hammer M, Blonde L, et al. Patient-reported outcomes following treatment with the human GLP1 analogue liraglutide or glimepiride in monotherapy: results from a randomized controlled trial in patients with type 2 diabetes. Diabetes Obes Metab 2010; 12: 604-12.

22 Russell-Jones D, Vaag A, Schmitz O, Sethi BK, Lalic N, Antic S, et al. Liraglutide effect and action in diabetes 5 (LEAD-5) met+SU study group. Liraglutide vs insulin glargine and placebo in combination with metformin and sulfonylurea therapy in type 2 diabetes mellitus (LEAD5 met+SU): a randomised controlled trial. Diabetologia 2009; 52 :
23 Buse JB, Rosenstock J, Sesti G, Schmidt WE, Montanya E, Brett JH, et al. Liraglutide once a day versus exenatide twice a day for type 2 diabetes: a 26-week randomised, parallel-group, multinational, openlabel trial (LEAD-6). Lancet 2009; 374: 39-47.

24 Marre M, Shaw J, Brändle M, Bebakar WM, Kamaruddin NA, Strand $\mathrm{J}$, et al. Liraglutide, a once-daily human GLP-1 analogue, added to a sulphonylurea over 26 weeks produces greater improvements in glycaemic and weight control compared with adding rosiglitazone or placebo in subjects with type 2 diabetes (LEAD-1 SU). Diabet Med 2009; 26: 268-78.

25 Zinman B, Gerich J, Buse JB, Lewin A, Schwartz S, Raskin P, et al. Efficacy and safety of the human glucagon-like peptide- 1 analog liraglutide in combination with metformin and thiazolidinedione in patients with type 2 diabetes (LEAD- 4 Met+TZD). Diabetes Care 2009; 32: 1224-30.

26 Klein S, Sheard NF, Pi-Sunyer X. Weight management through lifestyle modification for the prevention and management of type 2 diabetes: rationale and strategies. Diabetes Care 2004; 27: 2067-73.

27 Yajnik CS, Yudkin JS. The Y-Y paradox. Lancet 2004; 363: 163.

28 Dagenais GR, Yi Q, Mann JF. Prognostic impact of body weight and abdominal obesity in women and men with cardiovascular disease. Am Heart J 2005; 149: 54-60.

29 Fadini GP, Simioni N, Frison V, Dal Pos M, Bettio M, Rocchini P, et al. Independent glucose and weight-reducing effects of liraglutide in a real-world population of type 2 diabetic outpatients. Acta Diabetol 2013; 50: 943-9.

30 Park J, Mehrotra R, Rhee CM, Molnar MZ, Lukowsky LR, Patel SS, et al. Serum creatinine level, a surrogate of muscle mass, predicts mortality in peritoneal dialysis patients. Nephrol Dial Transplant 2013; 28: 2146-55.

31 Mamtani M, Kulkarni H, Dyer TD, Almasy L, Mahaney MC, Duggirala R, et al. Waist circumference independently associates with the risk of insulin resistance and type 2 diabetes in mexican american families. PLoS One 2013; 8: e59153.

32 Jendle J, Nauck MA, Matthews DR, Frid A, Hermansen K, Düring M, et al. Weight loss with liraglutide, a once-daily human glucagon-like peptide- 1 analogue for type 2 diabetes treatment as monotherapy or added to metformin, is primarily as a result of a reduction in fat tissue. Diabetes Obes Metab 2009; 11: 1163-72.

33 Garber A1, Henry R, Ratner R, Garcia-Hernandez PA, Rodriguez-Pattzi $\mathrm{H}$, Olvera-Alvarez I, et al. Liraglutide versus glimepiride monotherapy for type 2 diabetes (LEAD-3 Mono): a randomised, 52-week, phase III, double-blind, parallel-treatment trial. Lancet 2009; 373: 473-81.

34 Heritier SR, Gebski VJ, Keech AC. Inclusion of patients in clinical trial analysis: the intention-to-treat principle. Med J Aust 2003; 179: 438-40.

35 Hollis S, Campbell F. What is meant by intention to treat analysis. Survey of published randomised controlled trials? BMJ 1999; 319: 670-4. 